\title{
AMENDMENTS
}

\section{Author Correction: The kinase TBK1 controls IgA class switching by negatively regulating noncanonical NF-кB signaling}

Jin Jin, Yichuan Xiao, Jae-Hoon Chang, Jiayi Yu, Hongbo Hu, Robyn Starr, George C. Brittain, Mikyoung Chang, Xuhong Cheng and Shao-Cong Sun

Correction to: Nature Immunology https://doi.org/10.1038/ni.2423, published online 30 September 2012.

In the version of this article initially published, in Fig. 5a, the flow cytometry plot that is second from the right in the bottom row (+LPS, + TGF- $\beta+$ APRIL, $+\alpha$ CD40; NIK ${ }^{\mathrm{KO}}$ mice) did not display the correct data. The error occurred when the figure was converted from color to black and white; no changes were made to the data. The original and corrected Fig. $5 \mathrm{a}$ are shown below. The error has not been corrected in the original article.
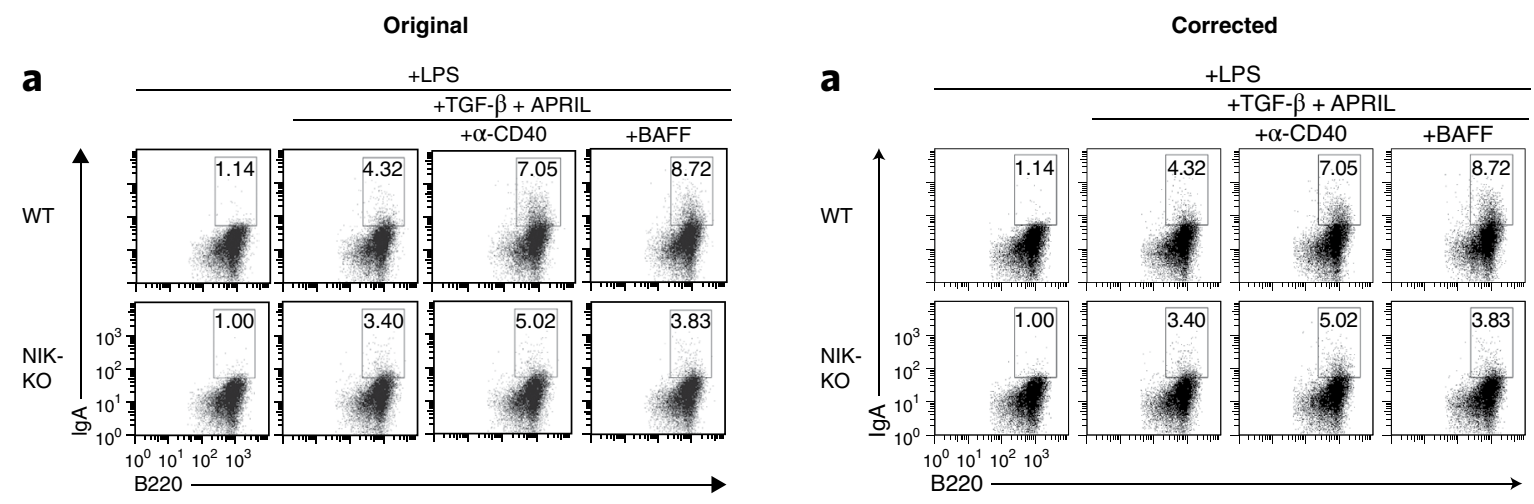

Fig. 5a | Original and corrected.

Published online: 3 July 2020

https://doi.org/10.1038/s41590-020-0748-8

๑) The Author(s), under exclusive licence to Springer Nature America, Inc. 2020

\section{Author Correction: The immunology of blood: connecting the dots at the neurovascular interface}

Katerina Akassoglou (D)

Correction to: Nature Immunology https://doi.org/10.1038/s41590-020-0671-z, published online 23 June 2020.

In the version of this article initially published, in the Acknowledgements, it was incorrectly stated that the author receives support from the Edward and Pearl Fein Trust. The author receives support from Edward and Pearl Fein. The error has been corrected in the HTML and PDF versions of the article.

Published online: 7 July 2020

https://doi.org/10.1038/s41590-020-0752-z

(C) The Author(s), under exclusive licence to Springer Nature America, Inc. 2020 